\title{
Feature-based multiple models improve classification of mutation-induced stability changes
}

\author{
Lukas Folkman ${ }^{1,2^{*}}$, Bela Stantic ${ }^{1}$, Abdul Sattar ${ }^{1,2}$ \\ From SNP-SIG 2013: Identification and annotation of genetic variants in the context of structure, function, \\ and disease \\ Berlin, Germany. 19 July 2013
}

\begin{abstract}
Background: Reliable prediction of stability changes in protein variants is an important aspect of computational protein design. A number of machine learning methods that allow a classification of stability changes knowing only the sequence of the protein emerged. However, their performance on amino acid substitutions of previously unseen non-homologous proteins is rather limited. Moreover, the performance varies for different types of mutations based on the secondary structure or accessible surface area of the mutation site.

Results: We proposed feature-based multiple models with each model designed for a specific type of mutations. The new method is composed of five models trained for mutations in exposed, buried, helical, sheet, and coil residues. The classification of a mutation as stabilising or destabilising is made as a consensus of two models, one selected based on the predicted accessible surface area and the other based on the predicted secondary structure of the mutation site. We refer to our new method as Evolutionary, Amino acid, and Structural Encodings with Multiple Models (EASE-MM). Cross-validation results show that EASE-MM provides a notable improvement to our previous work reaching a Matthews correlation coefficient of 0.44 . EASE-MM was able to correctly classify $73 \%$ and $75 \%$ of stabilising and destabilising protein variants, respectively. Using an independent test set of 238 mutations, we confirmed our results in a comparison with related work.
\end{abstract}

Conclusions: EASE-MM not only outperformed other related methods but achieved more balanced results for different types of mutations based on the accessible surface area, secondary structure, or magnitude of stability changes. This can be attributed to using multiple models with the most relevant features selected for the given type of mutations. Therefore, our results support the presumption that different interactions govern stability changes in the exposed and buried residues or in residues with a different secondary structure.

\section{Background}

A non-synonymous single nucleotide polymorphism (SNP) in a coding region of DNA results in a single amino acid polymorphism (a mutation) in a protein sequence. The ability to predict how such an amino acid substitution affects the stability of a protein is an important aspect of computational protein design. Moreover, it has been shown that disease-associated protein variants are often characterised by mutation-induced stability

\footnotetext{
* Correspondence: lukas.folkman@griffithuni.edu.au

'Institute for Integrated and Intelligent Systems, Griffith University, Brisbane, Australia

Full list of author information is available at the end of the article
}

changes [1]. Therefore, an improved prediction of stability changes may help us deepen our understanding of the relationship between protein mutations and inherited diseases.

With the immense amounts of data about protein variants coming from the genome sequencing projects, computational methods, being fast and inexpensive, became convenient tools to study stability changes. These computational approaches can be categorised as energy-based and training-based methods. While energy-based methods use physical, statistical, or empirical energy functions to estimate the stability change from the protein's threedimensional structure [2-9], training-based methods are 
trained on the experimental data from the ProTherm database [10] employing machine learning algorithms [11-26]. Interestingly, a number of the training-based methods allow for a prediction knowing only the sequence of a protein [17-26]. Since there is a large gap between the number of known protein sequences and experimentally determined three-dimensional structures, we devote our interest to these sequence-based methods in this work.

While a number of the sequence-based methods were able to report a high prediction accuracy, the results from an assessment study showed that the performance of three evaluated methods was much lower on an independent test set [27]. There, only the mutations from new additions to the ProTherm database were used for testing. This finding correlates with the results reported in our recent work [26]. We found that the prediction performance of three methods in our comparison was relatively low when evaluated solely on mutations in proteins with low sequence similarity to the training set. To improve prediction performance on non-homologous proteins, we proposed a method based on evolutionary and structural encodings with amino acid parameters. While the new method was able to outperform related work, the analysis revealed that the performance for exposed residues was considerably lower than for buried ones. Similarly, mutations in coil residues appeared to be more difficult to predict than the ones in $\alpha$-helices and $\beta$-sheets.

In this work, we followed the observation that prediction performance differs among various types of mutations based on the accessible surface area or secondary structure. By employing feature selection, we built specialised feature-based multiple models, each dedicated to a specific type of mutations. Our results show that this methodology improves two-class prediction of stability changes. Moreover, a consensus approach combining two methods with multiple models (one based on the accessible surface area and the other on the secondary structure) yielded further improvements. Analysis of our results revealed that the new method delivers more balanced predictions than our previous work for mutations in residues with a different secondary structure and solvent accessibility as well as for different magnitudes of stability changes. Finally, our new method achieved a favourable performance in a comparison with related work using an independent test set of 238 mutations. We refer to the new method as Evolutionary, Amino acid, and Structural Encodings with Multiple Models (EASE-MM).

\section{Methods}

\section{Feature-based multiple models}

We built and compared four different machine learning methods for the two-class (stabilising and destabilising) prediction of stability changes. The first one (referred to as EASE- $\mathrm{AA}_{2}$ ) was an extension of our previous work
(EASE-AA: Evolutionary And Structural Encodings with Amino Acid parameters [26]). EASE-AA 2 employed a single support vector machine (SVM) model with predictive features selected using a greedy feature selection algorithm. We used a range of features describing evolutionary conservation, amino acid parameters, and structural properties. Next, we developed two methods, each composed of several SVM models. The motivation here was to make each model specialised for different types of mutations. The first method (EASE-ASA) was composed of two models based on different accessible surface area (ASA) categories (exposed and buried). The second method (EASE-SS) was composed of three models based on different secondary structure (SS) types ( $\alpha$-helix, $\beta$-sheet, and coil). Finally, we built a consensus method combining the predicted probabilities of the two methods with multiple models. We refer to the consensus method as EASE-MM.

Figure 1 illustrates the design of EASE-SS, however, the same applies to EASE-ASA. First, we partitioned the training data according to the secondary structure (accessible surface area) of the mutation site. Second, we used feature selection to select a relevant combination of features for the given data partition. Next, the SVM parameters were optimised and the model was trained for classification of stability changes. Since we aimed to design strictly sequence-based methods, the secondary structure and two categories of accessible surface area were predicted with SPINE-X [28] and ACCpro [29], respectively. A solvent accessibility threshold of $25 \%$ was used for classifying residues as exposed or buried. This threshold resulted in a well-balanced partitions of 785 exposed and 891 buried mutations. Further discussion on the solvent accessibility thresholds can be found in the next section (Results and discussion).

Figure 2 illustrates how a prediction is performed using EASE-SS (the same applies to EASE-ASA). Given the

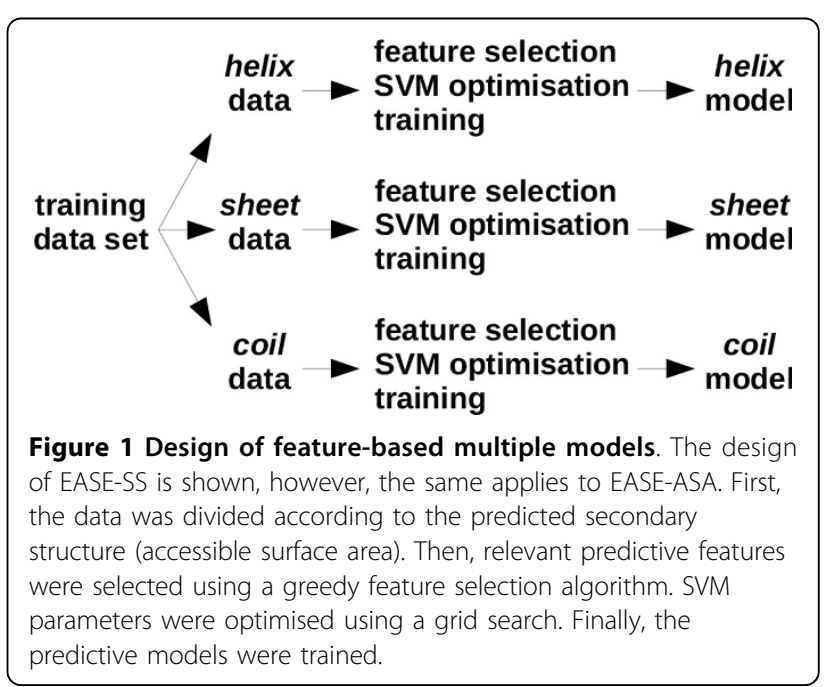




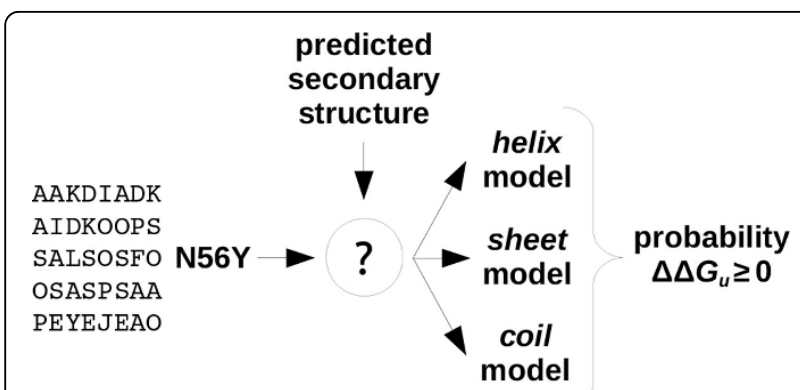

Figure 2 Classification with feature-based multiple models. The prediction process is shown for EASE-SS, however, the same applies to EASE-ASA. First, the secondary structure (accessible surface area) is predicted. Next, the relevant model is selected and used for prediction. That is, if the mutation is in a helical (exposed) residue, only the helix (exposed) model is used.

inputs (protein sequence and amino acid substitution), the secondary structure (accessible surface area) is predicted first. Then, the relevant model is selected. That is, if the mutation is in a helical (exposed) residue, only the helix (exposed) model is used. The output is a predicted probability of the mutation to be stabilising. Finally, for the case of the consensus method (not shown in the figure), prediction probability $P$ is the average of the probabilities predicted with EASE-ASA and EASE-SS:

$$
P_{\text {EASE }-M M}=\frac{1}{2}\left(P_{\text {EASE-ASA }}+P_{\text {EASE-SS }}\right)
$$

\section{Predictive features}

For machine learning classification of stability changes, each mutation needs to be encoded with a number of predictive features. We considered a range of features describing the evolutionary conservation, amino acids parameters, and structural properties.

\section{Evolutionary features}

We used two evolutionary features: SIFT score (also employed in our previous work $[25,26])$ and the difference of mutation and conservation likelihood $(\Delta M)$. SIFT [30] predicts whether a mutation affects the function of a protein. It is calculated from a scaled probability matrix of possible amino acid substitutions generated from a multiple sequence alignment of related sequences. SIFT scores range from 0 to 1 where scores below 0.05 are predicted as deleterious mutations. We ran SIFT using the SwissProt and TrEMBL databases with sequences more than $90 \%$ identical to the query removed.

Feature $\Delta M$ expresses the difference of likelihoods of the introduced and deleted amino acids to appear in the alignment of homologous sequences of the target protein. To calculate this feature, three iterations of PSI-BLAST [31] in default configuration were used to search the NCBI non-redundant database. Then, the likelihood scores were extracted from the last position specific scoring matrix
(PSSM). The scores were divided by 10 for normalisation so that most values fell within the range of -1 and 1 .

\section{Amino acid parameters}

A variety of different amino acid parameters were introduced for the prediction of stability changes $[12,14,16$, 20,23 ]. In our previous work [26], we adopted seven representative parameters including hydrophobicity, volume, polarisability, isoelectric point, helix tendency, sheet tendency, and a steric parameter (graph shape index). These parameters were first introduced in [32] and later applied to prediction of secondary structure [28]. In this work, we included another 4 parameters: flexibility [33], compressibility, bulkiness, and equilibrium constant with reference to the ionisation property of $\mathrm{COOH}$ group [34]. We included these parameters because they were found as one of the best determinants to stability changes in the study of 48 physical-chemical, energetic, and conformational amino acid properties [12,14]. We normalised all 11 parameters to fall within the range of -0.9 and 0.9 . The normalised values of the 11 parameters are available in Additional file 1.

We encoded each of the amino acid parameters as two distinct predictive features. The first one was equal to the difference between the amino acid parameters for the introduced and deleted amino acids (denoted as $\Delta$ ). The other predictive feature described the mutation site environment as the mean, minimum, and maximum of the parameter values for a window of six neighbouring residues. We considered neighbourhood windows of up to a length of 18 and found that six neighbours performed optimally.

\section{Structural features}

Since structural information is not available in the case of sequence-based prediction of stability changes, we employed predicted structural features. We used the multistep neural network method SPINE-X [28] for the prediction of secondary structure probabilities. Also, the real value of the relative accessible surface area of each mutation site was predicted with SPINE-X. For the prediction of the disorder probability, we used the neural network method SPINE-D [35]. These three predicted structural features were also used in our previous work [25,26].

\section{Feature selection}

We considered a range of predictive features and applied feature selection to design specialised models for 1) exposed and buried residues (EASE-ASA), and 2) helical, sheet, and coil residues (EASE-SS). Also, the single-model method (EASE- $\mathrm{AA}_{2}$ ) was designed employing the same feature selection procedure. We used sequential forward floating selection (SFFS) [36] which is a variation of a commonly adopted sequential forward selection (SFS) [37]. SFS works by iteratively adding the best-performing feature to a set of features $S$. Initially, $S$ is empty. Every iteration, the best-performing feature $f$ is selected as the one for which 
$S \cup\{f\}$ achieves the highest prediction performance. The SFFS algorithm starts with SFS but at the end of every iteration, features are iteratively removed if this can further improve prediction performance. Thus, the number of features in $S$ is not monotonously increasing because the search is 'floating' up and down. We ran SFFS until 10 features were added to $S$ and stored all visited combinations of features. Finally, we selected the ultimately best-performing of the visited combinations. As a measure of prediction performance, the area under the receiver operating characteristic curve (commonly denoted as AUC) was used

To verify the significance of the features selected with SFFS, we performed feature selection also with the stability selection algorithm [38]. In stability selection, the data sample of size $n$ is randomly sub-sampled to size $n / 2$. Then, an 'inner' feature selection algorithm is applied. The whole procedure is repeated $k$-times, each time with a different data sample. Features which are repeatedly selected contribute towards the final set of predictive features. We used SFS as the 'inner' algorithm and set $k=100$.

\section{Support vector machines}

Support vector machines (SVM) [39] are machine learning algorithms which can approximate non-linear functions by mapping the inputs to a high-dimensional feature space using a kernel function and then, solving a linear problem by finding a maximum margin separating hyperplane. We used the radial basis kernel function and implemented our method with the LIBSVM library [40].

To optimise the SVM performance, the regularisation parameter $C$ and the radial basis kernel width parameter $\gamma$ need to be set. If the number of positive and negative examples in the data set is unbalanced, SVM can be further optimised by setting the weight $(w)$ of the penalty for a training error on positive examples. We optimised these SVM parameters by running a grid search using 10 -fold cross-validation. In the grid search, we considered all possible combinations of $C \in\left\{2^{-5}, 2^{-3}, \ldots, 2^{7}\right\}$, $\gamma \in\left\{2^{-7}, 2^{-5}, \ldots, 2^{1}\right\}$, and $w \in\{1,1.5,2,2.5,3\}$.

\section{Data sets}

We compiled a data set of free energy stability changes from the ProTherm database [10] (February 2013). There, a stability change is defined as the difference in the unfolding free energy: $\Delta \Delta G_{u}\left[\mathrm{kcal} \mathrm{mol}^{-1}\right]=\Delta G_{u}($ mutant $)-\Delta G_{u}$ (wild-type). Hence, we designated the positive and negative examples of the classification problem as the stabilising $\left(\Delta \Delta G_{u} \geq 0\right)$ and destabilising $\left(\Delta \Delta G_{u}<0\right)$ mutations, respectively.

We extracted 3,329 mutations with listed stability changes and cross-checked all the sources where the measurements came from. We found that incorrect values (mostly the sign of $\Delta \Delta G_{u}$ ) had been entered from at least
18 sources. We corrected stability changes for all relevant (> 230) mutations in the extracted data set. Next, we removed all duplicate entries of the same amino acid substitutions (different concentrations of chemicals, stability changes of the protein intermediate state, etc.). If several measurements of the same mutation under the same experimental conditions were present, we averaged the stability changes and kept only a single entry. If several measurements of the same mutation under different experimental conditions were present, we kept only the measurement closest to the physiological $\mathrm{pH} 7$.

Finally, we identified 74 clusters of proteins with more than $25 \%$ sequence similarity using BLASTCLUST [41]. If there were several measurements of the same amino acid substitution within a single cluster, we kept only the measurement closest to the physiological pH 7. This process yielded a non-redundant data set containing 1,914 mutations of 95 different proteins grouped into 74 nonhomologous clusters.

To perform an independent comparison with related work, we separated all proteins with less than $25 \%$ sequence similarity to the data set used for the training of I-Mutant2.0 [17]. This procedure yielded 25 proteins with 238 mutations which we kept as an independent test set (S238). The remaining data of 1,676 mutations in 70 different proteins (S1676) were used for the development of our methods. Both S238 and S1676 data sets are available in Additional file 2.

\section{Evaluation}

We used unseen-protein 10-fold cross-validation on the S1676 data set to design our methods, select relevant features, and perform a comparison with our previous work (EASE-AA) [26]. In the unseen-protein cross-validation, we ensured that all mutations of any cluster of homologous proteins were contained within a single fold. Also, we kept the ratio of stabilising and destabilising examples reasonably similar among the folds. We repeated our experiments 100 times (each time with randomly generated folds) and averaged the results.

The unseen-protein cross-validation was previously used for the evaluation of a method for the prediction of deleterious mutations [42]. A similar procedure, unseenresidue cross-validation, was used for the evaluation of a three-state stability changes prediction method [21]. In our recent work [26], we compared both unseen-residue and unseen-protein cross-validation with the commonlyused random cross-validation. There, we concluded that the unseen-protein cross-validation provides the most robust estimate of the prediction performance. This is because predictive features cannot be selected as 'proxies' to specific residues or proteins.

For an independent comparison with related work, we used the S238 data set. This data was not used for the 
SVM parameters optimisation nor feature selection. Importantly, the sequence similarity between S1676 and S238 was less than $25 \%$. To achieve a fair comparison with related work, we optimised prediction thresholds of all compared methods to yield a maximum Matthews correlation coefficient (MCC). MCC is a measure of prediction performance that provides more relevant information than classification accuracy $\left(\mathrm{Q}_{2}\right)$ in cases when the data set is severely biased towards one class of examples. Since destabilising mutations prevail in the available experimental data, $72 \%$ and $81 \%$ of mutations were destabilising in the S1676 and S238 data sets, respectively.

Regarding evaluation measures, we assessed the overall prediction performance in terms of the receiver operating characteristic (ROC) curves and the area under the ROC curve (AUC). A ROC curve plots the true positive rate (sensitivity) as a function of the false positive rate (100\% - specificity) at different prediction thresholds. Furthermore, we calculated Matthews correlation coefficient $(\mathrm{MCC})$, classification accuracy $\left(\mathrm{Q}_{2}\right)$, sensitivity $(\mathrm{Se})$, specificity $(\mathrm{Sp})$, positive predictive value (PPV), and negative predictive value (NPV):

$$
\begin{aligned}
& \mathrm{MCC}=\frac{T P \times T N-F P \times F N}{\sqrt{(T P+F P)(T P+F N)(T N+F P)(T N+F N)}} \\
& \mathrm{Q}_{2}=\frac{T P+T N}{T P+F P+T N+F N} \times 100 \\
& \mathrm{Se}=\frac{T P}{T P+F N} \times 100 \\
& \mathrm{Sp}=\frac{T N}{T N+F P} \times 100 \\
& \mathrm{PPV}=\frac{T P}{T P+F P} \times 100 \\
& \mathrm{NPV}=\frac{T N}{T N+F N} \times 100,
\end{aligned}
$$

where $T P, T N, F P$, and $F N$ refer to the number of true positives, true negatives, false positives, and false negatives, respectively.

\section{Results and discussion}

Our main interest was to asses whether a method with feature-based multiple models can improve prediction performance compared to methods employing only a single model. To explore different ideas, we designed two methods with multiple models: EASE-ASA (composed of two models for exposed and buried residues) and EASE-SS (composed of three models for helical, sheet, and coil residues). We also built a consensus method (EASE-MM) of the former two. The most relevant features for each model were chosen using sequential forward floating selection (SFFS). We compared the 10-fold cross-validation performance (data set S1676) of the three methods with multiple models and two single-model methods (EASE-AA and EASE-AA ${ }_{2}$ ). While EASE-AA is our previous work [26], EASE- $\mathrm{AA}_{2}$ was designed using the same feature selection procedure as the methods with multiple models. Next, we analysed the significance of the selected features and investigated performance of our methods for different types of mutations. Finally, we performed a comparison with related work on an independent test set of 238 mutations.

\section{Cross-validation performance}

We performed 100 replications of 10-fold cross-validation on the S1676 data set. Table 1 summarises the averaged results. Our previous work, EASE-AA, yielded the lowest prediction performance with a Matthews correlation coefficient $(\mathrm{MCC})$ of 0.35 . EASE- $\mathrm{AA}_{2}$ achieved only a marginal absolute improvement of 0.03 in $\mathrm{MCC}$ reaching a value of 0.38 . We observed more notable improvements for the methods employing multiple models. EASE-ASA and EASE-SS reached MCC values of 0.40 and 0.42 , respectively. These results constitute respective relative improvements of $14 \%$ and $20 \%$ (absolute improvements of 0.05 and 0.07 ) compared to our previous work (EASE-AA). The consensus method combining the predicted probabilities of EASE-ASA and EASE$S S$ yielded further improvements. EASE-MM achieved an MCC of 0.44 which represents a relative improvement of 26\% (an absolute improvement of 0.09) compared to EASE-AA. Compared to EASE-AA 2 , the relative (absolute) improvement was $16 \%$ (0.06). While EASE-MM did not improve on EASE-AA, 's specificity (the accuracy on negative examples), negative predictive value was improved. This means that EASE-MM did not 'over-predict' destabilising mutations as much as EASE- $\mathrm{AA}_{2}$

The receiver operating characteristic (ROC) curves in Figure 3 compare the true positive rate of the five methods as a function of the false positive rate at different

Table 1 Cross-validation performance (data set S1676) of our previous work, the single-model method, and the three methods with multiple models.

\begin{tabular}{llllllll}
\hline Method & AUC & MCC & $\mathbf{Q}_{\mathbf{2}}$ & Se & Sp & PPV & NPV \\
\hline EASE-AA & 0.76 & 0.35 & 67.11 & 74.93 & 64.22 & 43.68 & 87.37 \\
EASE-AA $_{2}$ & 0.77 & 0.38 & 72.67 & 65.54 & 75.31 & 49.58 & 85.51 \\
EASE-ASA & 0.80 & 0.40 & 72.62 & 71.07 & 73.19 & 49.55 & 87.23 \\
EASE-SS & 0.80 & 0.42 & 73.57 & 72.13 & 74.11 & 50.79 & 87.77 \\
EASE-MM & 0.82 & 0.44 & 74.71 & 73.14 & 75.28 & 52.30 & 88.33 \\
\hline
\end{tabular}

EASE-MM is a consensus method of EASE-ASA and EASE-SS 


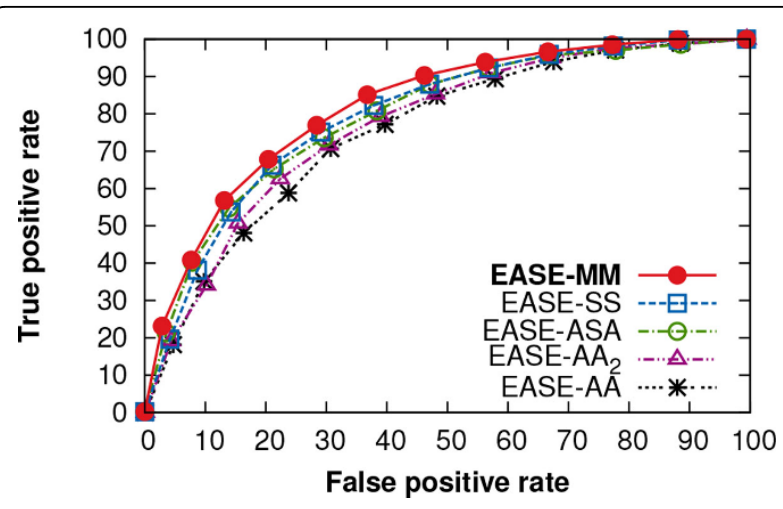

Figure 3 ROC curves performance of the three methods with multiple models, the single-model method, and our previous work. The true positive rate is shown as a function of the false positive rate at different prediction thresholds. These are crossvalidation results with the S1676 data set. EASE-MM, EASE-SS, EASE-ASA, EASE-AA ${ }_{2}$, and EASE-AA achieved the area under the ROC curve $(A \cup C)$ of $0.82,0.80,0.80,0.77$ and 0.76 , respectively.

prediction thresholds. The figure demonstrates the benefit in terms of the number of correctly predicted positive examples upon employing the methods with multiple models. EASE-ASA, EASE-SS, and EASE-MM achieved the area under the ROC curve (AUC) of 0.80 , 0.80 , and 0.82 , while EASE-AA and EASE- $\mathrm{AA}_{2}$ yielded an AUC of 0.76 and 0.77 , respectively. This represents a relative improvement of $6 \%$ (an absolute improvement of 0.05 ) for EASE-MM compared to EASE-AA 2 .

We estimated the statistical significance of the improvements yielded by the methods with multiple models over the 100 replications of cross-validation using a student $t$-test. The null hypothesis stated that there was no statistical difference in the MCC (AUC) for EASEMM (EASE-ASA, EASE-SS) compared to EASE-AA 2 (EASE-AA). The $p$-values associated with this null hypothesis were all less than 0.0001. Also, EASE-MM's improvements compared to EASE-ASA (EASE-SS) were statistically significant $(p$-values $<0.0001)$.

\section{Significance of the selected predictive features}

We used the sequential forward floating selection (SFFS) to automatically select the combinations of the most relevant predictive features. While seven and nine features were selected for the exposed and buried models of EASE-ASA, respectively, each model of EASE-SS (helix, sheet, and coil) was composed of eight features. Figure 4 shows the area under the ROC curve (AUC) as a function of the number features selected with the SFFS algorithm. This figure illustrates the motivation for our work well. While at the early stages of feature selection, there is a notable gap between the prediction performance for mutations in the exposed and buried residues, as feature selection progresses, the performance becomes balanced

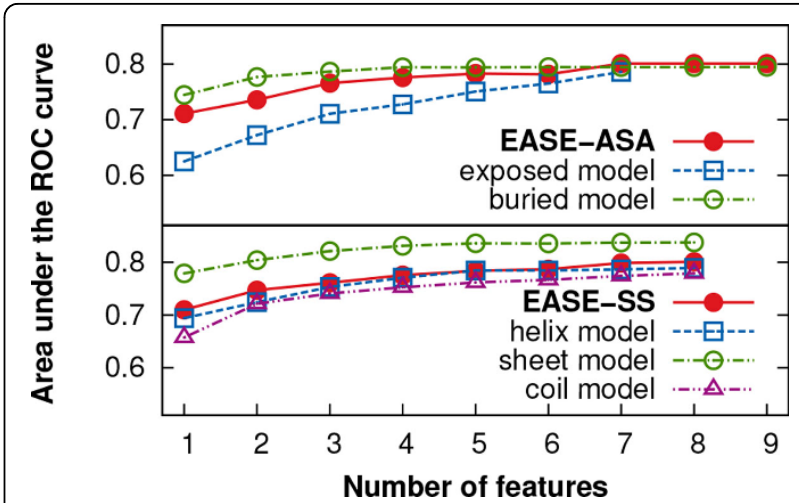

Figure 4 Feature selection progress. The area under the $\mathrm{ROC}$ curve $(A \cup C)$ is shown as a function of the number features selected during feature selection. The prediction performance of the different models became more balanced as the feature selection progressed. These are cross-validation results with the $\$ 1676$ data set.

for both categories. A similar trend, however not as pronounced, can be seen for the case of EASE-SS. Additional file 3 lists the final combinations of features implemented in EASE-ASA, EASE-SS, and EASE-AA .

We analysed which features were most often selected across the five different models of EASE-ASA and EASESS. SFFS selected feature SIFT score for each of the five models. Feature $\Delta$ hydrophobicity was selected in all but the sheet model. The third most often occurring feature was the relative accessible surface area. It is of interest to inspect which features were the most specific for each of the models. For instance, amino acid attributes $\Delta$ helix tendency and $\Delta$ sheet tendency were chosen for the helix and sheet models, respectively. These features are indeed relevant specifically to the two models because they express the change in the preference of the given secondary structure type. Feature $\Delta$ volume was selected for both helix and sheet but not for the coil model. We conjecture that an increase in the side-chain volume may induce strain in the backbone of the protein in regions with a well-defined secondary structure but can be better tolerated in a coil region. Regarding the two models of EASEASA, features $\Delta$ isoelectric point and $\Delta$ polarisability were selected for the buried but not for the exposed model.

While SFFS was effective in finding the most relevant combination of predictive features, it does not provide a ranking of the individual features. This is because, as the feature selection progresses, even a significant feature can be removed if it does not perform well in combination with the others. We employed stability selection to analyse the significance of individual features. We implemented stability selection as 100 replications of the basic sequential forward selection (SFS) each time executed on a randomly sub-sampled S1676 data set (data was not partitioned for different types of mutations in this 
experiment). From the 100 results, we estimated the significance of each feature as the probability of being selected. Table 2 lists the nine most significant features. Evolutionary feature SIFT score, amino acid parameter $\Delta$ hydrophobicity, and structural property relative accessible surface area seem to be the most significant. This finding agrees with the most often occurring features across the five models of EASE-ASA and EASE-SS.

\section{Evaluation of different types of mutations}

The design of the three methods combining feature-based multiple models was motivated by the observation that the prediction performance of our previous work (EASE-AA) varied considerably for different types of mutations. We found that EASE-ASA (combining two models), EASE-SS (three models), and EASE-MM (consensus of the former two) can predict stability changes more accurately than both single-model methods EASE-AA and EASE-AA (Figure 3 ). Next, we were interested what the improvements were for different types of mutations based on the secondary structure and accessible surface area of the mutated residue. Secondary structure and solvent accessibility were calculated using DSSP [43] from the experimentally determined structures deposited in the Protein Data Bank (PDB) [44]. We also investigated prediction performance for 'small' $\left(\Delta \Delta G_{u} \in[-1,1]\right)$ and 'large' $\left(\left|\Delta \Delta G_{u}\right|>1\right)$ stability changes.

Figure 5 compares the cross-validation performance (MCC) of EASE-AA 2 , EASE-ASA, EASE-SS, and EASE$M M$ for different types of mutations. We analysed the performance of the methods with multiple models relative to EASE- $\mathrm{AA}_{2}$ because while EASE- $\mathrm{AA}_{2}$ encompasses only a single model, it was designed using the same feature selection procedure. Also, EASE- $\mathrm{AA}_{2}$ performed marginally better than EASE-AA.

Regarding the different secondary structure types, EASEMM achieved an MCC of $0.49,0.42$, and 0.37 for the mutations in helical, sheet, and coil residues, respectively.

Table 2 The nine most significant features according to stability selection on the $\mathbf{S 1 6 7 6}$ data set.

\begin{tabular}{ll}
\hline Feature & Significance \\
\hline SIFT score & 1.00 \\
$\Delta$ hydrophobicity & 0.97 \\
relative accessible surface area & 0.86 \\
disorder probability & 0.81 \\
$\Delta$ compressibility & 0.80 \\
$\Delta$ polarisability & 0.58 \\
volume (mean, min, max) & 0.54 \\
$\Delta$ isoelectric point & 0.53 \\
secondary structure probabilities & 0.47
\end{tabular}

$\Delta$ refers to the change between the introduced and deleted amino acids; (mean, min, max) was calculated for a window of six neighbouring residues; significance denotes the probability of being selected with stability selection
The respective relative (absolute) improvements compared to EASE- $\mathrm{AA}_{2}$ were $11 \%$ (0.05), $2 \%$ (0.01), and 48\% (0.12). All four methods yielded the lowest performance for the category of coil residues. However, both relative and absolute improvements of all three methods with multiple models were the highest in this category. Thus, the methods with multiple models yielded a more balanced performance for the different secondary structure types than the single-model method. This is most apparent from the performance of EASE-SS which achieved an MCC of $0.43,0.40$, and 0.39 for helical, sheet, and coil residues, respectively.

Next, we analysed our results for two categories of accessible surface area (ASA) based on a threshold of $25 \%$. We found again that EASE-MM not only outperformed EASE- $\mathrm{AA}_{2}$ but achieved a more balanced performance yielding an MCC of 0.44 and 0.41 for residues with ASA $\leq$ $25 \%$ and $>25 \%$, respectively. These results constitute respective relative (absolute) improvements of $5 \%(0.02)$ and $37 \%(0.11)$ compared to EASE-AA 2 . The performance of EASE-MM in Figure 5 appears to be well balanced when we analysed only the two categories of ASA employing a threshold of $25 \%$ (the same threshold as for the design of the two models of EASE-ASA). Therefore, we were interested whether the performance varied if we considered a greater variety of ASA categories. Figure 6 shows the MCC of the compared methods as a function of four categories of ASA. The figure reveals that the performance of EASE-MM for residues more than $60 \%$ exposed to a solvent is on average $63 \%$ lower than for the other three categories covering ASA of $0-60 \%$. While the performance of EASE-ASA was also very low in the $>60 \%$ exposed category (an MCC of 0.05), there was a considerable improvement in the $20-40 \%$ category. Thus, it seems that the feature selection for the EASE-ASA's exposed model selected mainly features which are relevant to 'partially exposed' residues. This is likely because residues with ASA $>60 \%$ contribute only to $7 \%$ of the S1676 data set. For the same reason (the lack of experimental data), it would not be possible to design EASE-ASA with three models including a model trained specifically for residues with ASA $>60 \%$. An alternative way of improving the consensus method (EASE-MM) would be assigning a higher weight to the predicted probability by EASE-SS for mutations in residues predicted as $>60 \%$ exposed to a solvent. EASE-SS yielded an MCC of 0.25 in this ASA category.

Finally, we analysed performance for 'small' $\left(\Delta \Delta G_{u} \in\right.$ $[-1,1])$ and 'large' $\left(\left|\Delta \Delta G_{u}\right|>1\right)$ stability changes (Figure 5). For all four methods, the category of 'small' changes was more difficult to predict. EASE-MM reached an MCC of 0.36 and 0.48 for the 'small' and 'large' stability changes, respectively. The reason for this unbalanced performance can be twofold. Firstly, it is naturally harder to differentiate among subtle changes. Secondly, 


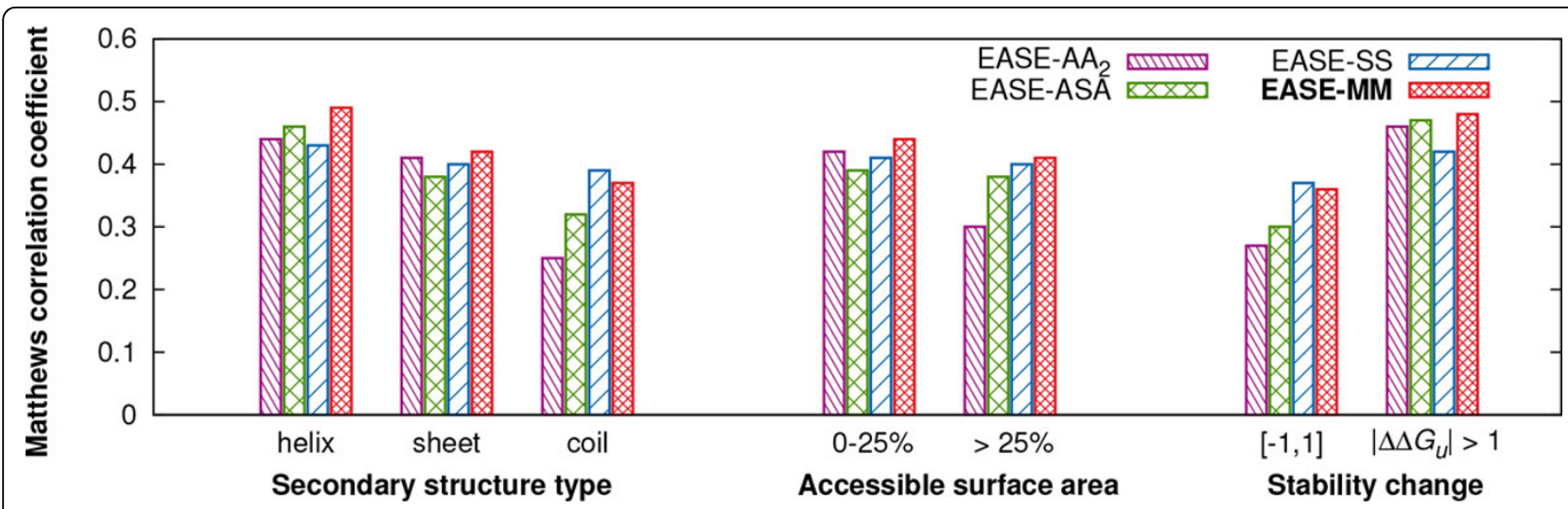

Figure 5 Prediction performance of the single-model method and the three methods with multiple models for different types of mutations. Matthews correlation coefficient (MCC) of EASE-AA 2 , EASE-ASA, EASE-SS, and EASE-MM is shown as a function of the secondary structure type of the mutated residue, relative accessible surface area of the mutated residue, and magnitude of the stability change. These are cross-validation results with the $\mathbf{S 1 6 7 6}$ data set.

as suggested elsewhere [21,20], the strict classification of the 'small' stability changes as stabilising or destabilising can be misleading since the experimental data is affected by the error of measurement which can be as large as $\pm 0.48 \mathrm{kcal} \mathrm{mol}^{-1}$ [45]. Nevertheless, the relative (as well absolute) improvement of EASE-MM (compared to EASE- $\mathrm{AA}_{2}$ ) was larger for the 'small' stability changes (33\% while it was $4 \%$ for the 'large' stability changes).

Overall, EASE-ASA, EASE-SS, and EASE-MM yielded a more balanced performance for all categories of different types of mutations than the single-model method EASE-AA 2 . This result supports our hypothesis that building specialised models for different types of mutations can yield a more balanced performance. When comparing the performance of the consensus method

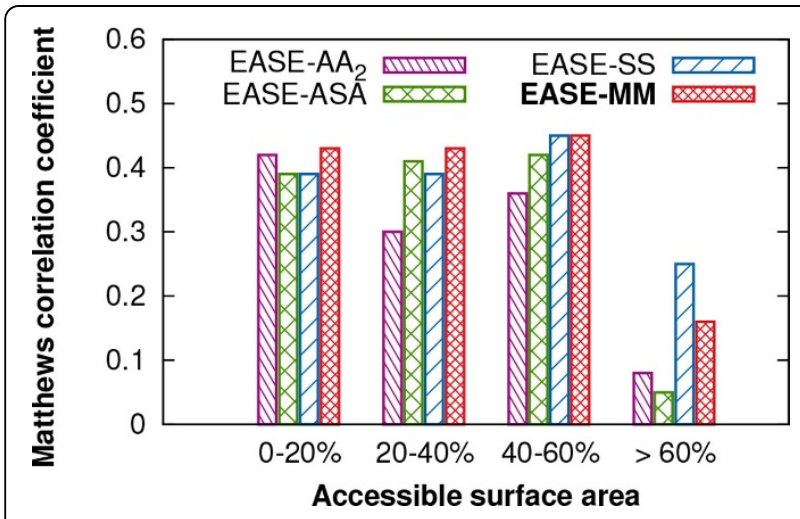

Figure 6 Prediction performance of the single-model method and the three methods with multiple models for different categories of solvent accessibility. Matthews correlation coefficient (MCC) of EASE-AA ${ }_{2}$ EASE-ASA, EASE-SS, and EASE-MM is shown as a function of four different categories of mutated residue's relative accessible surface area. These are cross-validation results with the S1676 data set.
(EASE-MM) with EASE-ASA, we found improvements in all seven categories (Figure 5). However, compared to EASE-SS, EASE-MM performed less accurately for mutations in coil residues and for 'small' stability changes. This can be attributed to relatively low performance of EASE-ASA for these two types of mutations. Because the predicted probabilities from EASE-ASA and EASE-SS contribute equally to predictions made by EASE-MM, a low performance of one of the two methods directly influences the consensus. A possible improvement would be to consider the confidence of the predicted secondary structure and use it for weighing the contribution of the probabilities predicted with EASE-SS and EASE-ASA.

\section{Independent test performance}

We found that EASE-MM yielded the highest cross-validation prediction performance of the five compared methods (Figure 3). However, it is important to inspect its prediction performance on an independent test set to see if the feature selection did not result in features which do not generalise well. Using the S238 data set, we compared the performance of three currently available methods (MUpro [18], MuStab [23], and IMutant2.0 [17]), our previous work (EASE-AA [26]), the single-model method (EASE- $\mathrm{AA}_{2}$ ), and the three methods with multiple models (EASE-ASA, EASE-SS, and EASE-MM). Table 3 summarises the results from the independent comparison. EASE-MM was able to considerably outperform the three currently available methods. The absolute increase in the MCC ranged from 0.20 to 0.23. The ROC curves in Figure 7 compare the true positive rate as a function of the false positive rate at different prediction thresholds. The absolute improvements in terms of the AUC for EASE-MM compared to MUpro, MuStab, and I-Mutant2.0 were 0.20, 0.18, 0.15, 
Table 3 Independent test performance (data set S238) of three currently available methods, our previous work, the single-model method, and the three methods with multiple models.

\begin{tabular}{llllllll}
\hline Method & AUC & $\mathbf{M C C}$ & $\mathbf{Q}_{\mathbf{2}}$ & Se & Sp & PPV & NPV \\
\hline MUpro & 0.65 & 0.24 & 79.41 & 28.89 & 91.19 & 43.33 & 84.62 \\
MuStab & 0.67 & 0.26 & 77.31 & 40.00 & 86.01 & 40.00 & 86.01 \\
I-Mutant2.0 & 0.70 & 0.27 & 65.97 & 68.89 & 65.28 & 31.63 & 90.00 \\
EASE-AA & 0.83 & 0.45 & 82.35 & 60.00 & 87.56 & 52.94 & 90.37 \\
EASE-AA $_{2}$ & 0.72 & 0.36 & 82.77 & 35.56 & 93.78 & 57.14 & 86.19 \\
EASE-ASA $^{0.81}$ & 0.43 & 83.19 & 51.11 & 90.67 & 56.10 & 88.83 \\
EASE-SS & 0.82 & 0.48 & 83.19 & 62.22 & 88.08 & 54.90 & 90.91 \\
EASE-MM & 0.85 & 0.47 & 81.09 & 68.89 & 83.94 & 50.00 & 92.05 \\
\hline
\end{tabular}

EASE-MM is a consensus method of EASE-ASA and EASE-SS

respectively. The performance of the three currently available methods was in agreement with the findings reported in our previous work [26]. There, we described how the evaluation is influenced when different mutations of proteins from the training set are used for testing. Since sequence similarity of the S238 data set and the data used for developing MUpro, MuStab, and I-Mutant 2.0 was less than $25 \%$, the performance of these three methods was rather low.

The three methods with multiple models (EASE-ASA, EASE-SS, EASE-MM) yielded the AUC (MCC) of 0.81 (0.43), $0.82(0.48)$, and $0.85(0.47)$, respectively (Table 3 ). All three methods were able to considerably outperform the single-model method EASE-AA 2 . However, when compared to our previous work (EASE-AA), only EASE$\mathrm{MM}$ was able to improve the AUC value (from 0.83 to 0.85). Figure 7 compares EASE-MM and EASE-AA in terms of ROC curves. For the false positive rate of $15-45 \%$, our new method achieved a notable improvement.

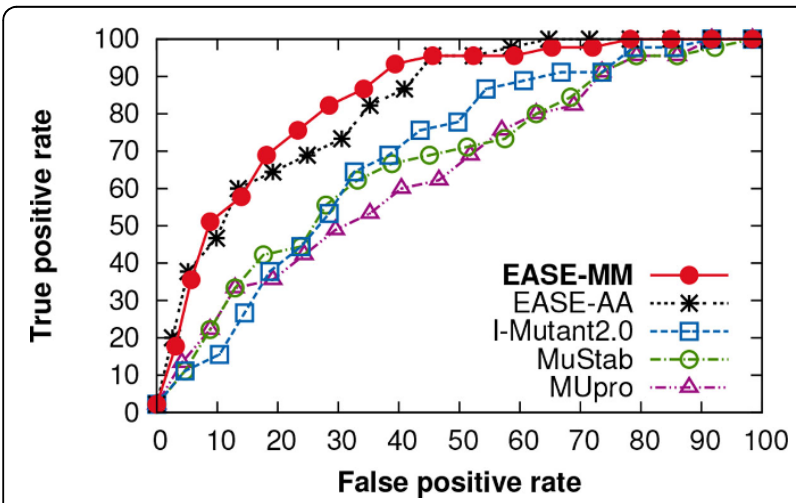

Figure 7 ROC curves performance of EASE-MM, our previous work, and three currently available methods. The true positive rate is shown as a function of the false positive rate at different prediction thresholds. These are independent test results with the S238 data set. EASE-MM, EASE-AA, I-Mutant2.0, MuStab, and MUpro achieved the area under the ROC curve (AUC) of $0.85,0.83,0.70$, 0.67 and 0.65 , respectively.
This is reflected in a $2 \%$ and $4 \%$ relative increase in the AUC and MCC, respectively.

We analysed why the improvements to our previous work (EASE-AA) on the S238 test set (Table 3) were not as high as for the cross-validation on the S1676 data set (Table 1). We found that there was a relative decrease of $19 \%$ in MCC on residues with accessible surface area (ASA) $\leq 25 \%$ for EASE-MM compared to EASE-AA. Coincidently, the accuracy of the predicted accessible surface area with ACCpro (decides which of the two models of EASE-ASA would be used) was 5 percentage points lower for the buried residues [a decrease from $85 \%$ (S1676) to 80\% (S238)]. We conjecture that this might be one of the contributing factors to the relatively low prediction accuracy of EASE-MM on the residues with ASA $\leq 25 \%$. For the residues with ASA > 25\%, EASE-MM provided a relative improvement of $50 \%$ which is in good agreement with a $46 \%$ improvement yielded in cross-validation. However, the abundance of the residues with ASA > 25\% was considerably lower in S238 (34\%) compared to S1676 (48\%). Therefore, despite the improvement for the $>25 \%$ exposed residues, the overall relative increase in EASE-MM's MCC was only $4 \%$ on the S238 data set.

To confirm the significance of the improvements yielded by our new method on the S238 test set, we randomly sub-sampled the data to 119 mutations and classified the stability changes with EASE-MM and EASE-AA. We replicated this experiment 100 times. The student $t$-test's null hypothesis stated that there was no statistical difference in the MCC (AUC) between EASE-MM and EASE-AA. The $p$-values associated with this null hypothesis were less than 0.0001 for both MCC and AUC.

\section{Conclusions}

In this work, we followed the observation that the prediction performance of our previous work [26] varies for different types of mutations based on the accessible surface area and secondary structure. We proposed a sequencebased machine learning method, EASE-MM, which predicts stability changes as a consensus of predicted probabilities of two participating methods, EASE-ASA and EASE-SS. While EASE-ASA combines two models for exposed and buried residues, EASE-SS is composed of three models for mutations in $\alpha$-helices, $\beta$-sheets, and coils. Feature selection and a range of diverse features were used to design each model.

Our cross-validation results show that EASE-MM provides a notable improvement to our previous work reaching a Matthews correlation coefficient of 0.44 (Table 1). EASE-MM was able to correctly classify $73 \%$ and $75 \%$ of stabilising and destabilising protein variants, respectively, and yielded the area under the ROC curve of 0.82 (Figure 3). Using an independent test set of 238 
mutations, we confirmed our results in a comparison with related work (Figure 7).

EASE-MM not only outperformed our previous work and other related methods, it achieved a more balanced results for different types of mutations based on the accessible surface area, secondary structure, and magnitude of stability changes (Figures 5 and 6). This can be attributed to using multiple models with the most relevant features selected for the given type of mutations. Therefore, our results support the presumption that different interactions govern stability changes in the exposed and buried residues [8] or in residues with a different secondary structure. Similar observations have been made about pathogenic protein variants [46]. Therefore, we aim to extend the concept of the feature-based models to prediction of diseaseassociated mutations [42,47-55] in our future work.

\section{Availability of supporting data}

The data sets supporting the results of this article are included within the article and its additional files. The source code of our method is available for download from www.ict.griffith.edu.au/bioinf/ease.

\section{Additional material}

Additional file 1: Amino acid parameters. The file containing the values of the 11 normalised amino acid parameters is available in a white-space-delimited tabular text format.

Additional file 2: Data sets S1676 and S238. The files containing both the $\mathbf{S 1 6 7 6}$ and S238 data sets are available in a white-space-delimited tabular text format. All files are compressed in a single zip archive.

Additional file 3: Final combinations of predictive features. The list of the final combinations of predictive features implemented in the single model of EASE-AA 2 , two models of EASE-ASA, and three models of EASE-SS is available in PDF format.

\section{Competing interests}

The authors declare that they have no competing interests.

\section{Authors' contributions}

LF designed the study, developed the methods, and conducted the data analysis under the guidance of BS. LF drafted the manuscript. BS and AS contributed to the manuscript preparation.

\section{Acknowledgements \\ Helpful discussion with Yaoqi Zhou and Yuedong Yang is gratefully acknowledged. NICTA is funded by the Australian Government as represented by the Department of Broadband, Communications and the Digital Economy, and the Australian Research Council through the ICT Centre of Excellence program.}

\section{Declarations}

The publication costs for this article were funded by the Institute for Integrated and Intelligent Systems, Griffith University, and NICTA. This article has been published as part of BMC Genomics Volume 15 Supplement 4, 2014: SNP-SIG 2013: Identification and annotation of genetic variants in the context of structure, function, and disease. The full contents of the supplement are available online at http://www.biomedcentral.com/ bmcgenomics/supplements/15/S4

\section{Authors' details}

${ }^{1}$ Institute for Integrated and Intelligent Systems, Griffith University, Brisbane, Australia. ${ }^{2} \mathrm{NICTA}$ - National ICT Australia.

Published: 20 May 2014

\section{References}

1. Laskowski RA, Thornton JM: Understanding the molecular machinery of genetics through 3D structures. Nature Reviews Genetics 2008, 9(2):141-151.

2. Zhou H, Zhou Y: Distance-scaled, finite ideal-gas reference state improves structure-derived potentials of mean force for structure selection and stability prediction. Protein Science 2002, 11(11):2714-2726.

3. Guerois R, Nielsen JE, Serrano L: Predicting changes in the stability of proteins and protein complexes: A study of more than 1000 mutations. Journal of Molecular Biology 2002, 320(2):369-387.

4. Bordner A, Abagyan R: Large-scale prediction of protein geometry and stability changes for arbitrary single point mutations. Proteins: Structure, Function, and Bioinformatics 2004, 57(2):400-413.

5. Yin S, Ding F, Dokholyan NV: Eris: An automated estimator of protein stability. Nature Methods 2007, 4(6):466-467.

6. Deutsch C, Krishnamoorthy B: Four-body scoring function for mutagenesis. Bioinformatics 2007, 23(22):3009.

7. Benedix A, Becker CM, de Groot BL, Caflisch A, Bockmann RA: Predicting free energy changes using structural ensembles. Nature Methods 2009, 6(1):3-4.

8. Dehouck Y, Grosfils A, Folch B, Gilis D, Bogaerts P, Rooman M: Fast and accurate predictions of protein stability changes upon mutations using statistical potentials and neural networks: PoPMuSiC-2.0. Bioinformatics 2009, 25(19):2537.

9. Kellogg EH, Leaver-Fay A, Baker D: Role of conformational sampling in computing mutation-induced changes in protein structure and stability. Proteins: Structure, Function, and Bioinformatics 2011, 79:830-838.

10. Kumar M, Bava KA, Gromiha MM, Prabakaran P, Kitajima K, Uedaira H, Sarai A: ProTherm and ProNIT: Thermodynamic databases for proteins and proteinnucleic acid interactions. Nucleic Acids Research 2006, 34(Suppl 1):204.

11. Capriotti E, Fariselli P, Casadio R: A neural-network-based method for predicting protein stability changes upon single point mutations. Bioinformatics 2004, 20(Suppl 1):63.

12. Huang LT, Saraboji K, Ho SY, Hwang SF, Ponnuswamy M, Gromiha MM: Prediction of protein mutant stability using classification and regression tool. Biophysical Chemistry 2007, 125(2-3):462-470.

13. Masso M, Vaisman II: Accurate prediction of stability changes in protein mutants by combining machine learning with structure based computational mutagenesis. Bioinformatics 2008, 24(18):2002.

14. Kang $S, C$ Chen $G$, Xiao G: Robust prediction of mutation-induced protein stability change by property encoding of amino acids. Protein Engineering Design and Selection 2009, 22(2):75.

15. Tian J, Wu N, Chu X, Fan Y: Predicting changes in protein thermostability brought about by singleor multi-site mutations. BMC Bioinformatics 2010, 11(1):370.

16. Yang $Y$, Chen B, Tan G, Vihinen M, Shen B: Structure-based prediction of the effects of a missense variant on protein stability. Amino Acids 2013, 44(3):847-855.

17. Capriotti E, Fariselli P, Casadio R: I-Mutant2.0: Predicting stability changes upon mutation from the protein sequence or structure. Nucleic Acids Research 2005, 33(Suppl 2):306-310.

18. Cheng J, Randall A, Baldi P: Prediction of protein stability changes for single-site mutations using support vector machines. Proteins: Structure, Function, and Bioinformatics 2006, 62(4):1125-1132.

19. Huang LT, Gromiha MM, Ho SY: iPTREE-STAB: Interpretable decision tree based method for predicting protein stability changes upon mutations. Bioinformatics 2007, 23(10):1292.

20. Shen B, Bai J, Vihinen M: Physicochemical feature-based classification of amino acid mutations. Protein Engineering Design and Selection 2008, 21(1):37-44.

21. Capriotti E, Fariselli P, Rossi I, Casadio R: A three-state prediction of single point mutations on protein stability changes. BMC Bioinformatics 2008, 9(Suppl 2):6.

22. Ozen A, Gonen M, Alpaydin E, Haliloglu T: Machine learning integration for predicting the effect of single amino acid substitutions on protein stability. BMC Structural Biology 2009, 9(1):66. 
23. Teng S, Srivastava A, Wang L: Sequence feature-based prediction of protein stability changes upon amino acid substitutions. BMC Genomics 2010, 11(Suppl 2):5.

24. Chen CW, Lin J, Chu YW: iStable: off-the-shelf predictor integration for predicting protein stability changes. BMC Bioinformatics 2013, 14(Suppl 2):5.

25. Folkman L, Stantic B, Sattar A: Sequence-only evolutionary and predicted structural features for the prediction of stability changes in protein mutants. BMC Bioinformatics 2013, 14(Suppl 2):6.

26. Folkman L, Stantic B, Sattar A: Towards sequence-based prediction of mutation-induced stability changes in unseen non-homologous proteins. BMC Genomics 2014, 15(Suppl 1):4.

27. Khan S, Vihinen MA: Performance of protein stability predictors. Human Mutation 2010, 1(1):675.

28. Faraggi E, Zhang T, Yang Y, Kurgan L, Zhou Y: SPINE X: Improving protein secondary structure prediction by multistep learning coupled with prediction of solvent accessible surface area and backbone torsion angles. Journal of Computational Chemistry 2012, 33(3):259-267.

29. Pollastri G, Baldi P, Fariselli $P$, Casadio R: Prediction of coordination number and relative solvent accessibility in proteins. Proteins: Structure, Function, and Bioinformatics 2002, 47(2):142-153.

30. $\mathrm{Ng}$ PC, Henikoff S: Predicting deleterious amino acid substitutions. Genome research 2001, 11(5):863-874.

31. Altschul SF, Madden TL, Schaffer AA, Zhang J, Zhang Z, Miller W, Lipman DJ: Gapped BLAST and PSI-BLAST: A new generation of protein database search programs. Nucleic Acids Research 1997, 25(17):3389.

32. Meiler J, Muller M, Zeidler A, Schmaschke F: Generation and evaluation of dimension-reduced amino acid parameter representations by artificial neural networks. Molecular modeling annual 2001, 7(9):360-369.

33. Vihinen $M$, Torkkila $E$, Riikonen P: Accuracy of protein flexibility predictions. Proteins: Structure, Function, and Bioinformatics 1994, 19(2):141-149.

34. Gromiha MM, Oobatake M, Kono H, Uedaira H, Sarai A: Relationship between amino acid properties and protein stability: buried mutations. Journal of Protein Chemistry 1999, 18(5):565-578.

35. Zhang T, Faraggi E, Xue B, Dunker AK, Uversky VN, Zhou Y: SPINE-D: Accurate prediction of short and long disordered regions by a single neural-network based method. Journal of Biomolecular Structure and Dynamics 2012, 29(4):799-813.

36. Pudil P, Novovicova J, Kittler J: Floating search methods in feature selection. Pattern Recognition Letters 1994, 15(11):1119-1125.

37. Whitney AW: A direct method of nonparametric measurement selection. IEEE Transactions on Computers 1971, 100(9):1100-1103.

38. Meinshausen N, Buhlmann P: Stability selection. Journal of the Royal Statistical Society: Series B (Statistical Methodology) 2010, 72(4):417-473.

39. Cortes C, Vapnik V: Support-vector networks. Machine Learning 1995 20(3):273-297

40. Chang CC, Lin CJ: LIBSVM: A library for support vector machines. $A C M$ Transactions on Intelligent Systems and Technology 2011, 2(3):27-12727.

41. Altschul SF, Gish W, Miller W, Myers EW, Lipman DJ: Basic local alignment search tool. Journal of Molecular Biology 1990, 215(3):403-410.

42. Adzhubei IA, Schmidt S, Peshkin L, Ramensky VE, Gerasimova A, Bork P, Kondrashov AS, Sunyaev SR: A method and server for predicting damaging missense mutations. Nature Methods 2010, 7(4):248-249.

43. Kabsch W, Sander C: Dictionary of protein secondary structure: pattern recognition of hydrogen-bonded and geometrical features. Biopolymers 1983, 22(12):2577-2637.

44. Berman HM, Westbrook J, Feng Z, Gilliland G, Bhat T, Weissig H, Shindyalov IN, Bourne PE: The protein data bank. Nucleic Acids Research 2000, 28(1):235-242.

45. Khatun J, Khare SD, Dokholyan NV: Can contact potentials reliably predict stability of proteins? Journal of Molecular Biology 2004, 336(5):1223-1238.

46. Khan $\mathrm{S}$, Vihinen M: Spectrum of disease-causing mutations in protein secondary structures. BMC Structural Biology 2007, 7(1):56.

47. Thomas PD, Kejariwal A: Coding single-nucleotide polymorphisms associated with complex vs. Mendelian disease: evolutionary evidence for differences in molecular effects. Proceedings of the National Academy of Sciences 2004, 101(43):15398-15403.

48. Capriotti E, Calabrese R, Casadio R: Predicting the insurgence of human genetic diseases associated to single point protein mutations with support vector machines and evolutionary information. Bioinformatics 2006, 22(22):2729-2734.
49. Yue P, Melamud E, Moult J: SNPs3D: candidate gene and SNP selection for association studies. BMC Bioinformatics 2006, 7(1):166.

50. Bromberg $Y$, Yachdav $G$, Rost B: SNAP predicts effect of mutations on protein function. Bioinformatics 2008, 24(20):2397-2398.

51. Thusberg J, Vihinen M: Pathogenic or not? And if so, then how? Studying the effects of missense mutations using bioinformatics methods. Human Mutation 2009, 30(5):703-714.

52. Li B, Krishnan VG, Mort ME, Xin F, Kamati KK, Cooper DN, Mooney SD, Radivojac P: Automated inference of molecular mechanisms of disease from amino acid substitutions. Bioinformatics 2009, 25(21):2744-2750.

53. Wainreb G, Ashkenazy H, Bromberg Y, Starovolsky-Shitrit A, Haliloglu T, Ruppin E, Avraham KB, Rost B, Ben-Tal N: MuD: an interactive web server for the prediction of non-neutral substitutions using protein structural data. Nucleic acids research 2010, 38(Suppl 2):523-528.

54. Zhao $H$, Yang $Y$, Lin H, Zhang $X$, Mort M, Cooper DN, Liu Y, Zhou Y: DDIGin: discriminating between disease-associated and neutral nonframeshifting micro-indels. Genome Biology 2013, 14(3):23.

55. Bendl J, Stourac J, Salanda O, Pavelka A, Wieben ED, Zendulka J, Brezovsky J, Damborsky J: PredictSNP: robust and accurate consensus classifier for prediction of disease-related mutations. PLoS Computational Biology 2014, 10(1):1003440.

doi:10.1186/1471-2164-15-S4-S6

Cite this article as: Folkman et al:: Feature-based multiple models improve classification of mutation-induced stability changes. BMC Genomics 2014 15(Suppl 4):S6.

\section{Submit your next manuscript to BioMed Central and take full advantage of:}

- Convenient online submission

- Thorough peer review

- No space constraints or color figure charges

- Immediate publication on acceptance

- Inclusion in PubMed, CAS, Scopus and Google Scholar

- Research which is freely available for redistribution
C Biomed Central 